\title{
Enhanced nonlinear spectral compression in fibre by external sinusoidal phase modulation
}

\author{
S Boscolo ${ }^{1}$, L Kh Mouradian ${ }^{2}$ and C Finot ${ }^{3}$ \\ ${ }^{1}$ Aston Institute of Photonic Technologies, School of Engineering and Applied Science, \\ Aston University, Birmingham B4 7ET, United Kingdom \\ ${ }^{2}$ Ultrafast Optics Laboratory, Faculty of Physics, Yerevan State University, Yerevan \\ 0025, Armenia \\ ${ }^{3}$ Laboratoire Interdisciplinaire Carnot de Bourgogne, UMR 6303 CNRS - Université de \\ Bourgogne-Franche-Comté, 9 avenue Alain Savary, BP 47870, 21078 Dijon Cedex, \\ France \\ E-mail : christophe.finot@u-bourgogne.fr
}

\begin{abstract}
We propose a new, simple approach to enhance the spectral compression process arising from nonlinear pulse propagation in an optical fibre. We numerically show that an additional sinusoidal temporal phase modulation of the pulse enables efficient reduction of the intensity level of the side lobes in the spectrum that are produced by the mismatch between the initial linear negative chirp of the pulse and the self-phase modulation-induced nonlinear positive chirp. Remarkable increase of both the extent of spectrum narrowing and the quality of the compressed spectrum is afforded by the proposed approach across a wide range of experimentally accessible parameters.
\end{abstract}

Keywords. Spectral compression, nonlinear propagation, optical fibre, phase modulation.

\section{Introduction}

The effect of optical nonlinearity on an ultra-short pulse propagating in an optical fibre is ordinarily associated with broadening of the pulse spectrum. In certain regimes, however, the fibre nonlinearity can induce the opposite effect, resulting in spectral compression of the pulse [1]. For example, a fundamental soliton propagating in an anomalous dispersion-increasing fibre can experience narrowing of its spectral width as a result of its adiabatic adaptation to the slowly varying fibre dispersion [2]. The working principle is the reverse operation of the well-known adiabatic soliton temporal compression in a dispersion-decreasing fibre [3]. Additionally, ultra-short laser pulses can be spectrally compressed in the regime of soliton self-frequency shift induced by the Raman effect in a highly nonlinear fibre [4]. Owing 
to the anomalous dispersion of the highly nonlinear fibre, laser pulses evolve toward solitons and experience a continuous frequency downshift. A lowering frequency and increasing dispersion of a redshifting soliton dictate spectral narrowing. Spectral narrowing can also be realized in an optical fibre by using self-phase modulation (SPM) of pulses with an initial negative frequency modulation (chirp) [5-7], such as the one imparted by an anomalously dispersive component. Indeed, the intensity-dependent nonlinear phase shift induced by SPM results, in silica, in a frequency downshift in the leading edge of the pulse and an upshift in the trailing edge. Thus the effect of SPM is dependent on the sign of the initial chirp. Negatively chirped pulses, where the long and the short wavelengths are in the trailing and the leading edges, respectively, are spectrally compressed, since both the long and the short wavelengths are shifted toward the center wavelength. This method of spectral compression has been implemented using various types of fibres [8-11], optical gain fibres, and photonic crystal fibres, and is suitable for a very large range of wavelengths including Ti:sapphire wavelengths $[8,9]$, the widely used $1-\mu \mathrm{m}[12,13]$ and $1.55-\mu \mathrm{m}[14]$ windows and the emerging $2-\mu \mathrm{m}$ band [15]. The process can also sustain simultaneous amplification of the pulse, and has been reported for different types of fiber amplifiers $[12,13,15,16]$. Therefore, spectral compression by SPM of negatively chirped pulses provides an attractive solution to convert ultra-short pulses delivered by femtosecond oscillators into powerful, near-transform-limited picosecond pulses, and to counteract the spectrum expansion that usually occurs with the direct amplification of picosecond structures. The concept can be extended to multistage architectures [17] and fibre laser cavities, where in-cavity nonlinear spectral compression in a mode-locked fibre laser has been recently demonstrated numerically [18]. However, the main limitation of SPM-driven spectral compression in the nonlinearity-dominant regime of propagation, in which fibre dispersion is of little importance, is the presence of residual side lobes in the compressed spectrum. These side lobes stem from the fact that in general an input pulse with a negative linear chirp cannot be compressed to the Fourier transform limit, because the cancellation of linear and nonlinear phases cannot in general be made complete. In order to overcome this limitation and, thus, approach a Fourier-transform-limited compression, several strategies have been proposed and experimentally demonstrated, including the use of complex phase modulation of the initial pulse to balance the (higher-order) dispersion and chirp [8] or the use of a pre-shaped input pulse profile such as a parabolic waveform [11, 14]. Another strategy to enhance the quality of the compressed pulse spectrum is to select a dispersive nonlinear regime of propagation in which the combined action of normal group-velocity dispersion (GVD) and SPM results in a deformation of the temporal profile of the pulse tending to acquire a rectangular shape while nearly complete compensation of the pulse chirp occurs $[19,20]$.

In this paper, we present a new method for enhancing the performance characteristics of the spectral compression by SPM of conventional laser pulses in a fibre, which is based on imposing an additional sinusoidal temporal phase modulation on the propagating pulse. We provide a thorough characterization of the compression process based on extensive numerical simulations. The optimal operational conditions are identified, which enables us to provide general design rules for spectralcompression fibre schemes over the parameter space that is typically accessible experimentally. Remarkably, our results show that high-quality compression with efficient reduction of the intensity level of spectral substructures is possible even for a spectrum that has split into a complex pattern. First, we introduce the situation being studied and the metrics used to quantify the performance of the compression process. Next we recall the results that can be obtained in the purely nonlinear propagation regime, and 
then we describe the effects of an additional temporal phase modulation of the pulse. Finally, we discuss the influence of the initial stretching ratio on the performances.

\section{Situation under investigation}

Our numerical simulations of the spectral compression process in a fibre system are based on the standard nonlinear Schrödinger equation (NLSE) [21]:

$$
i \frac{\partial \psi}{\partial z}+\gamma|\psi|^{2} \psi-\frac{1}{2} \beta_{2} \frac{\partial^{2} \psi}{\partial t^{2}}=0
$$

where $\psi(z, t)$ is the complex electric field envelope, $z$ is the propagation distance, $t$ is the reduced time, $\beta_{2}$ is the GVD parameter and $\gamma$ is the coefficient of cubic nonlinearity of the fibre. This equation neglects the effect of fibre loss, as well as higher-order linear and nonlinear effects. Although these effects can have noticeable impact on pulses shorter than $1 \mathrm{ps}$, here we neglect them as the leading-order behavior is well approximated by Eq. (1).

For the purpose of illustration, we consider a transform-limited pulse with a Gaussian intensity profile $\psi_{0}(t)=\sqrt{P_{0}} \exp \left(-t^{2} / 2 T_{0}^{2}\right)$ as the initial condition for our study. The parameters $T_{0}$ and $P_{0}$ are a characteristic temporal value (the half-width at $1 / e$-intensity point in the case of a Gaussian-shaped pulse) and the peak power of the initial pulse, respectively. The linear negative temporal chirp required for spectral compression to take place can be imprinted onto the initial pulse by stretching the pulse in the temporal domain in an anomalously dispersive medium, such as a pair of diffraction gratings [9, 16], a prism pair [6, 8], a fibre Bragg grating, or a segment of hollow core [12] or standard [14] fibre with anomalous GVD (and very low nonlinearity). As a result of GVD [described by Eq. (1) with $\gamma=0$ ], the different spectral components of the pulse acquire a frequency dependent delay. Even though such phase changes do not affect the pulse spectrum, they lead to temporal broadening of the pulse and a time dependence of the pulse phase. If $A$ denotes the temporal broadening factor, the peak power of the pulse will drop by the same factor $\left(P_{1}=P_{0} / A\right)$ owing to the conservation of energy (in the absence of losses). In the far-field regime (i.e., when $A \gg 1$ ), the temporal phase becomes parabolic, so that the pulse envelope at the exit of the dispersive element takes the form $\psi(0, t)=\left|\psi_{1}(t)\right| \exp \left(-i b t^{2}\right)$, where $\left|\psi_{1}(t)\right| \square \sqrt{P_{0} / A} \exp \left(-t^{2} / 2 A^{2} T_{0}^{2}\right)$ is the stretched amplitude profile and $b \square-1 / 2 A T_{0}^{2}<0$ is the chirp parameter. This negatively linearly chirped pulse is then spectrally compressed in a nonlinear fibre. Hereinafter, we consider the situation when the nonlinearity-dominant regime of propagation is applicable, which is typical of various demonstrations of spectral compression due to SPM in fibres reported so far $[9,14]$. In this regime, the dispersion term in Eq. (1) plays a relatively minor role and can be neglected. An in-depth analysis of the situation in which dispersion can impact the nonlinear propagation of pulses in a fibre has been presented in previous works $[19,20]$, and is beyond the scope of this paper.

The narrowing of the pulse spectrum is quantified with the spectral compression factor $C_{\mathrm{fwhm}}$ $\left(\mathrm{C}_{\mathrm{rms}}\right)$ defined as the ratio of the spectral full-width at half maximum (FWHM) [root-mean-square (rms)] width at some distance in the fibre and the entrance of the fibre. In order to assess the quality of the spectral compression, we use the Strehl ratio $S$ defined as the ratio of the maximum spectral brilliance of the actual pulse to the spectral brilliance obtained assuming a flat temporal phase of the pulse [14]. Therefore, $S$ is comprised between 0 and 1, with 1 defining an ideal compression. 


\section{Gaussian pulse evolution in a purely nonlinear medium}

The nonlinear term in the pulse-propagation equation (1), in the limit $\beta_{2}=0$, imposes a temporal intensity distribution-dependent phase shift on the initial pulse, so that after a propagation length $z$ :

$$
\psi(z, t)=e^{i \gamma|\psi(0, t)|^{2} z} \psi(0, t)=e^{i \gamma\left|\psi_{1}(t)\right|^{2} z-i b t^{2}}\left|\psi_{1}(t)\right|
$$

It is seen that the instantaneous frequency of the pulse,

$$
\delta v(z, t)=-\frac{1}{2 \pi} \frac{d \phi}{d t}=-\frac{\gamma}{2 \pi} \frac{d}{d t}\left(\left|\psi_{1}(t)\right|^{2}\right) z+\frac{1}{\pi} b t=\frac{t}{2 \pi A T_{0}^{2}}\left[\frac{2 z}{A^{2} L_{N L}} \exp \left(-\left(\frac{t}{A T_{0}}\right)^{2}\right)-1\right]
$$

cannot in general be made equal to zero for all times, which expresses the fact that an input Gaussian pulse (and by extension any other non-parabolic pulse [11, 14]) with a negative linear chirp cannot be spectrally compressed to the Fourier-transform limit. In Eq. (3), $L_{N L}=1 /\left(\gamma P_{0}\right)$ is the nonlinear length associated with the pulse at the input to the system. It is useful here to expand the Gaussian function of the pulse profile in a Taylor series about the pulse center, namely,

$$
\delta v(z, t) \square \frac{1}{2 \pi A T_{0}^{2}}\left\{\left(\frac{2 z}{A^{2} L_{N L}}-1\right) t-\frac{2 z}{A^{2} L_{N L}}\left(\frac{1}{A T_{0}}\right)^{2} t^{3}+\frac{z}{A^{2} L_{N L}}\left(\frac{1}{A T_{0}}\right)^{4} t^{5}-\frac{z}{3 A^{2} L_{N L}}\left(\frac{1}{A T_{0}}\right)^{6} t^{7}+\ldots\right\}
$$

The equation above shows that the linear part of the instantaneous frequency yields zero for all $t$ at the propagation distance $z_{1}$ given by [20]:

$$
\frac{z_{1}}{L_{N L}}=\frac{A^{2}}{2}
$$

However, the translation of the well-known time-space analogy [22] into the spectral domain [23] indicates that the higher-order chirp terms in (4) cause spectral aberrations.

An example of the pulse evolution along the nonlinear fibre is presented in Fig. 1 for the initial stretching factor $A=20$. Significant spectral compression occurs in the fibre, followed by splitting of the pulse spectrum into an increasing number of substructures [Fig. 1(a)]. The evolutions of parameters $C_{\text {fwhm, }}$ $C_{\text {rms }}$ and $S$ are given in Fig. 2. It is seen that the propagation distance at which the Strehl ratio reaches its maximum value (denoted by $z_{2}$ ) differs from the distance $z_{1}$ of chirp cancellation near the pulse center given by Eq. (5) [20]. We can also infer from Fig. 2 that the highest brilliance in the central region of the pulse spectrum is achieved at the point of maximum Strehl ratio. While compression factors above 10 can be achieved in terms of FWHM spectral width, the rms-width compression factor attains moderate values below 2. This is a signature of the presence of non-negligible pedestals in the pulse spectrum. These pedestals degrade the quality of the compression, as confirmed by the Strehl ratio's values that do not exceed 0.4. The abrupt drop of $C_{\text {fwhm }}$ after the distance at which it is at its maximum is due to an increase in the intensity level of spectral satellites above the $-3 \mathrm{~dB}$ threshold used for its computation. The pulse spectrum splits up shortly after the distance of maximum $C_{\mathrm{fwhm}}$. 

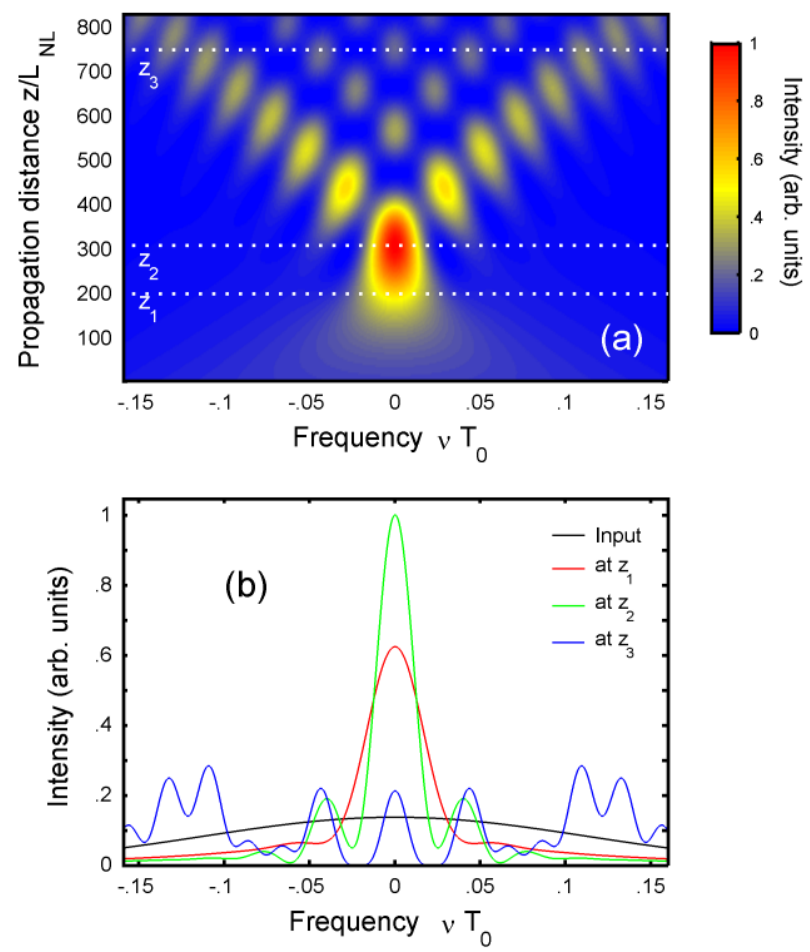

Figure 1 - Spectral compression of an initial Gaussian pulse with the stretching factor $A=20$ in a purely nonlinear fibre: (a) Longitudinal evolution of the spectral intensity profile. (b) Spectral intensity profile at different propagation distances in the fibre: the point $z_{1}$, predicted by Eq. (5) (red), the point $z_{2}$ of maximum Strehl ratio (green), and the point $z_{3}$ (blue). Also shown is the initial pulse profile (black).

In Fig. 1(b) we can see that at the distance $z_{1}$ foretold by Eq. (5) the pulse spectrum is effectively conveniently compressed, and the spectral profile does not display any oscillations, which is in agreement with the monotonic temporal variation of the frequency chirp at this distance (Fig. 3): the chirp continuously decreases across the pulse, and has a point of inflexion at the pulse center. At the other operational distances, the spectrum features an oscillating structure, which results from interference between different pulse parts having the same instantaneous frequency. This is confirmed by the nonmonotonic temporal variation of the chirp. Such a constructive interaction can have a beneficial impact on the spectral compression process, as it can increase the peak spectral intensity of the pulse with consequent enhancement of spectral brightness, as shown by the pulse spectrum obtained at the optimum compression point in terms of Strehl ratio, $z_{2}$. However, for propagation in the fibre beyond $z_{2}$, as a result of this interference phenomenon the pulse develops strong and detrimental oscillations in the wings together with strong spectral side lobes, as it appears from the spectrum at the distance $z_{3}=750 L_{N L}$. On the other hand, at $z_{1}$, while the spectrum narrowing in terms of FWHM spectral width is suboptimal, the spectrum exhibits significantly lower substructures than at further propagation distances. We note that this scenario is similar to the situation when the $B$-integral accumulation in chirped pulse amplification systems is not exactly compensated for: as a result of the distortion of the linear chirp by SPM, temporal structure unavoidably appears in the recompressed pulse, even for relatively small values of $B$ [22]. 


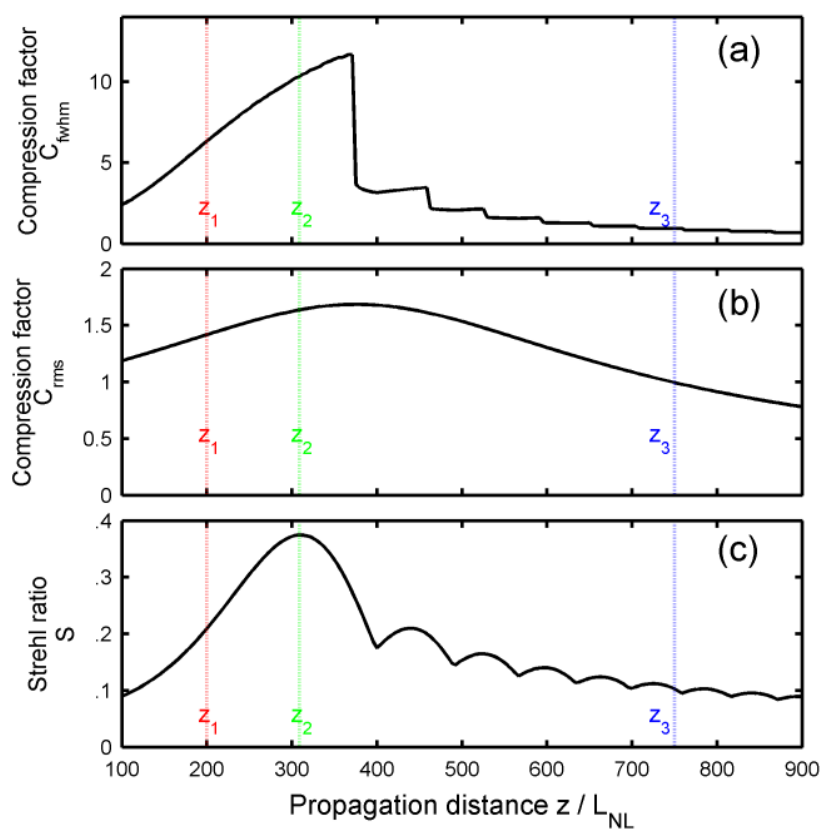

Figure 2 - Spectral compression of an initial Gaussian pulse with the stretching factor $A=20$ in a purely nonlinear fibre: longitudinal evolution of (a), (b) the FWHM and rms spectral compression factors, and (c) the Strehl ratio.

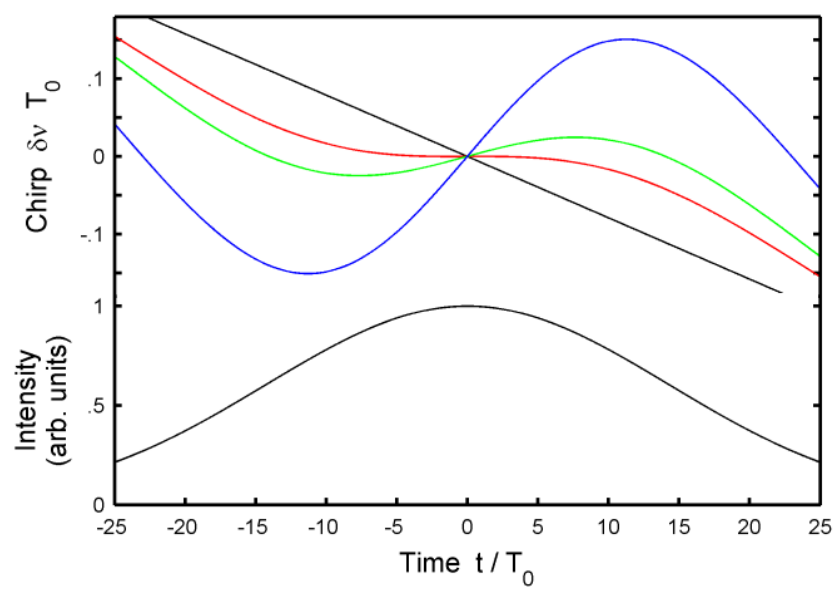

Figure 3 - Temporal intensity and chirp profiles of an initial Gaussian pulse with the stretching factor $A=20$ at different propagation distances in a purely nonlinear fibre: the point $z_{1}$, predicted by Eq. (5) (red), the point $z_{2}$ of maximum Strehl ratio (green), and the point $z_{3}$ (blue). Also shown are the initial pulse profiles (black).

\section{Use of an additional external phase modulation}

Now we describe the impact of an additional temporal phase modulation of the pulse on the spectral compression performances in the pure SPM case. Equation (5) gives the propagation distance at which the quadratic temporal phase (linear chirp) of the pulse is canceled, and Fig. 3 confirms at this distance the chirp is zero in the central part of the pulse. To compensate for the residual chirp, one could think of canceling the quartic and higher-order terms in the nonlinear phase stemming from the Taylor series expansion of the Gaussian profile (cubic and higher-order terms in Eq. (4)) by means of an external phase modulation. However, in practice, it may be quite difficult to synthetize such a complex phase profile in the temporal domain due to optoelectronic bandwidth limitations. Further, the validity of the Taylor 
expansion is rather limited to the central part of the pulse: for instance, an expansion to $o\left(t^{6}\right)$ would noticeably deviate from the actual phase of the pulse at times $|t| /\left(A T_{0}\right)$ greater than 0.5 . On the other hand, we can see in Fig. 3 that at the distance $z_{3}$, the chirp features strong oscillations with a close-to-sinusoidal variation in the central region of the pulse. This observation forms the basis of the simple idea proposed here to offset the chirp: apply to the pulse a corrective sinusoidal temporal phase modulation, $\phi_{a}(t)=-a_{c}$ $\cos \left(2 \pi v_{c} t\right)$, producing the chirp $\delta v_{a}(t)=a_{c} v_{c} \sin \left(2 \pi v_{c} t\right)$. Such a modulation can be imparted by a lithium-niobate phase modulator driven by a synchronized microwave signal [23-25] or by cross-phase modulation of the pulse with a sinusoidal signal [26]. The amplitude and frequency of the modulation can be intuitively chosen as $a_{c}=\Delta v \Delta T$ and $v_{c}=1 / 2 \Delta T$, where $\Delta T$ represents the time separation between the two local extrema of the chirp in the central region of the pulse at a given propagation distance $z>z_{1}$, and $\Delta v$ is the difference between the corresponding chirp values.

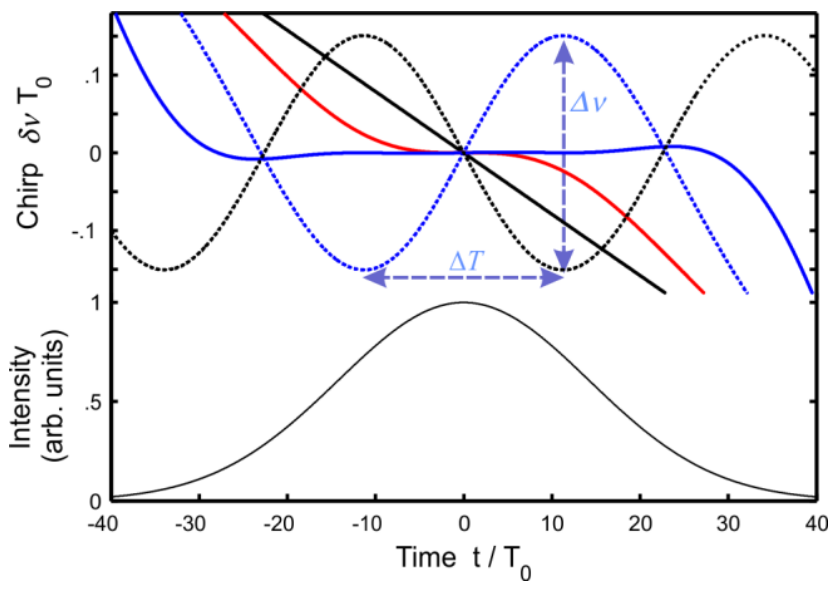

Figure 4 - Spectral compression of an initial Gaussian pulse with the stretching factor $A=20$ in a purely nonlinear fibre: temporal chirp profiles at the propagation distance $z_{3}$ before (dotted blue) and after (solid blue) phase correction. The corrective chirp $\delta v_{a}$ is plotted with a dotted black line. Also shown are the pulse characterizations at the entrance of the fibre (black), at the distance $z_{1}$, predicted by Eq. (5) (red) in the absence of phase correction.

Figure 4 illustrates this approach at the distance $z_{3}$. The chirp is indeed made linear and flat over most of the pulse (for $|t| / T_{0}<30$ ). On the contrary, the compensation of the linear chirp at $z_{1}$ leads to a significantly narrower region of chirp cancellation restricted to $|t| / T_{0}<10$. The resulting spectrum at $z_{3}$ (Fig. 5) highlights the striking enhancement of the spectral compression afforded by the corrective approach: even a very degraded spectrum can be corrected and changed to a high-quality compressed spectrum. Most of the pulse energy is concentrated in the central lobe of the spectrum while the intensity level of the side lobes is significantly lower than that occurring at $z_{1}$ or $z_{2}$ in the absence of phase correction [Fig. 5(b)], which entails a remarkable increase in spectral brightness or compression quality. 

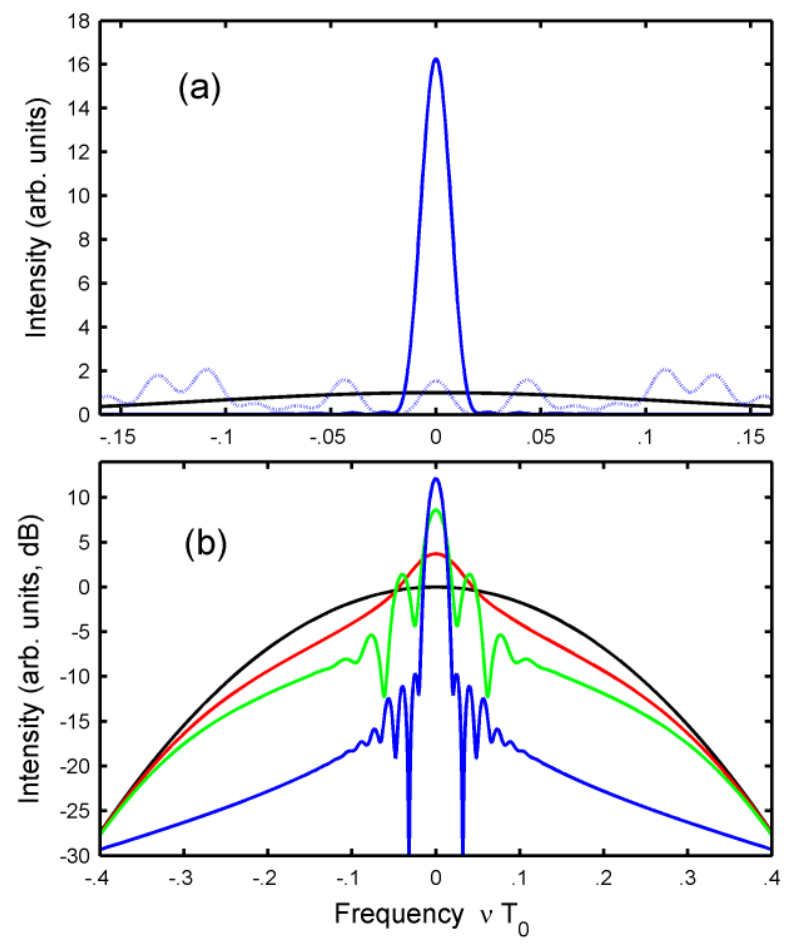

Figure 5 - Impact of phase correction on the spectral compression of an initial Gaussian pulse with the stretching factor $A=20$ in a purely nonlinear fibre: spectral intensity profiles plotted on (a) linear and (b) logarithmic scales at the propagation distance $z_{3}$ before (dotted blue) and after (solid blue) phase correction. Also shown are the profiles at the entrance of the fibre (black), at the distance $z_{1}$, predicted by Eq. (5) (red), and the distance $z_{2}$ of maximum Strehl ratio (green), in the absence of phase correction.
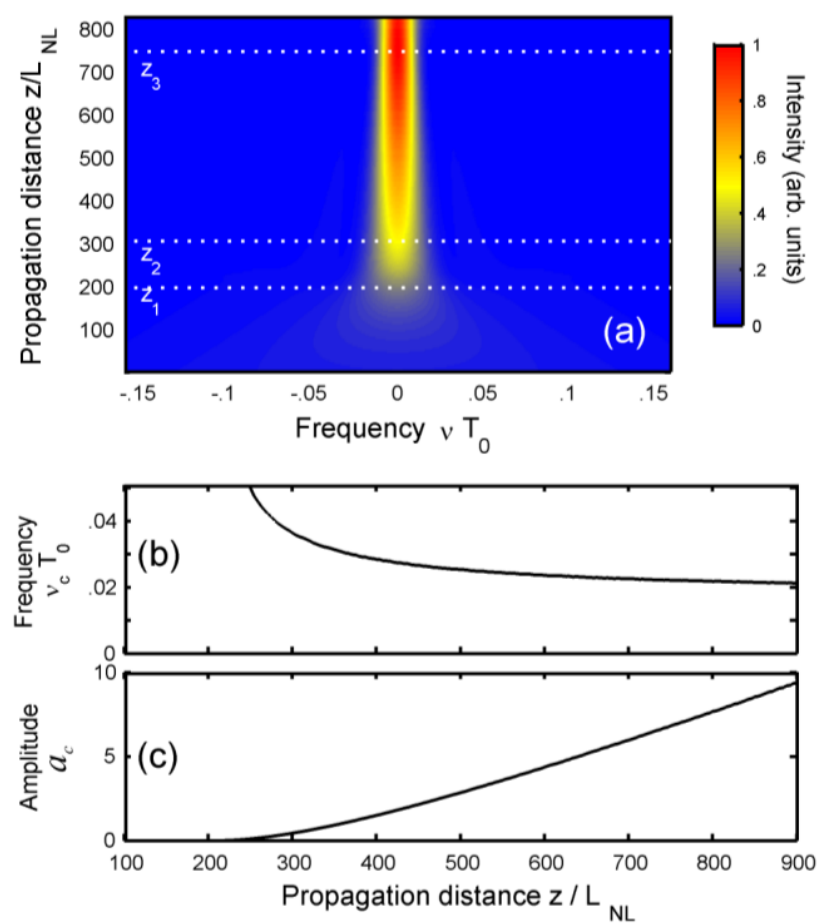

Figure 6 - Spectral compression of an initial Gaussian pulse with the stretching factor $A=20$ in a purely nonlinear fibre with phase correction: longitudinal evolution of (a) the spectral intensity profile of the pulse, and (b), (c) the frequency $v_{c}$ and amplitude $a_{c}$ of the corrective phase modulation. 
The enhancement of the spectral compression performance characteristics enabled by the corrective phase modulation method is in fact noticeable at any propagation distance in the fibre beyond $z_{2}$. Figures 6 and 7 summarize the results obtained using this approach. It is seen that the frequency of the corrective modulation decreases monotonically with increasing propagation distance, whereas the amplitude increases. The sinusoidal phase correction has little impact on the performances for propagation distances between $z_{1}$ and $z_{2}$, where the chirp remains nearly linear and flat near the pulse center. The strength of the approach increases with increasing propagation distance beyond $z_{2}$ as the pulse chirp develops an increasingly stronger sinusoidal-like temporal variation. The compression ratio is significantly increased with this strategy both in terms of FWHM and rms widths. The FWHM compression factor can now exceed 15. Remarkably, the rms compression factor and the Strehl ratio feature up to threefold and more than twofold increase, respectively, with respect to their maximum value in the absence of phase correction, confirming the efficient reduction of spectral wings and enhanced quality of the compression.
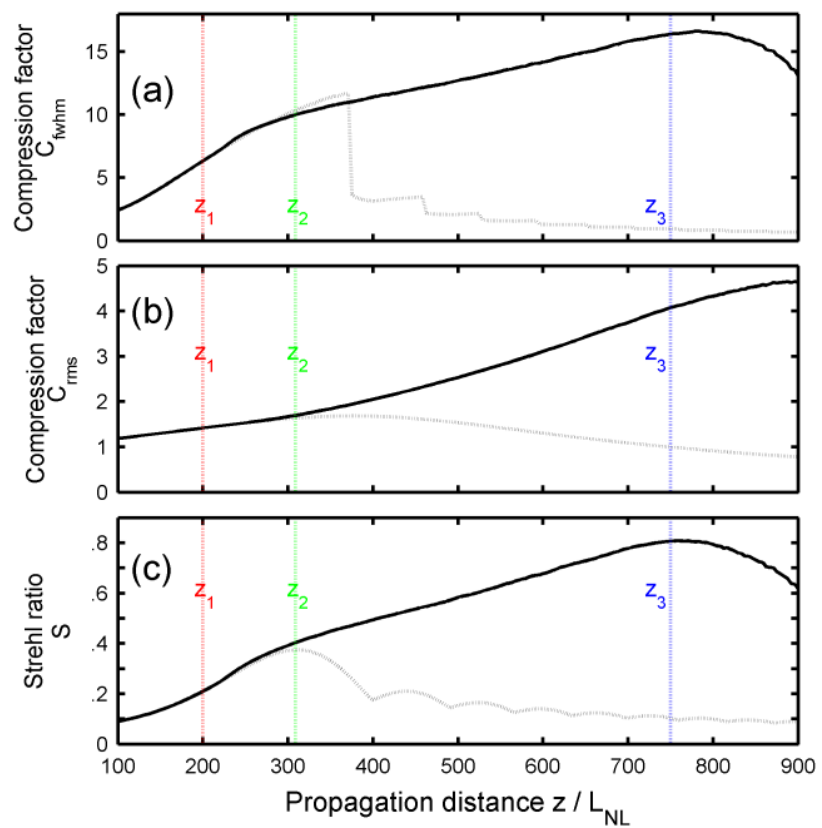

Figure 7 - Spectral compression of an initial Gaussian pulse with the stretching factor $A=20$ in a purely nonlinear fibre with phase correction: longitudinal evolution of (a), (b) the FWHM and rms spectral compression factors, and (c) the Strehl ratio. Also shown are the results obtained without correction (dotted grey).

We also studied the effect of the initial temporal broadening factor $A$ on the performance characteristics of the compression process. In the maps presented in Fig. 8, we summarize the results obtained using stretching values that are typical of experimental realizations [14]. We can note some common performance trends between the two cases of nonlinear propagation in the absence and in the presence of phase correction: larger $A$ results in a larger maximum FWHM compression factor, while the overall quality of the process rather deteriorates with increasing $A$, as indicated by the moderate decrease of the Strehl ratio [20]. A larger $A$ also leads to longer optimum propagation distances in terms of both compression factors and Strehl ratio. On the other hand, the application of a corrective sinusoidal phase 
modulation to the propagating pulse remarkably improves the compression process in both the achievable extent of spectrum narrowing and the quality of the compressed spectrum for all $A$ values being studied. Further, the use of the corrective approach enables significant broadening of the range of operational parameters that support performances near to the optimum, thus providing higher flexibility in the design of the spectral compression scheme.

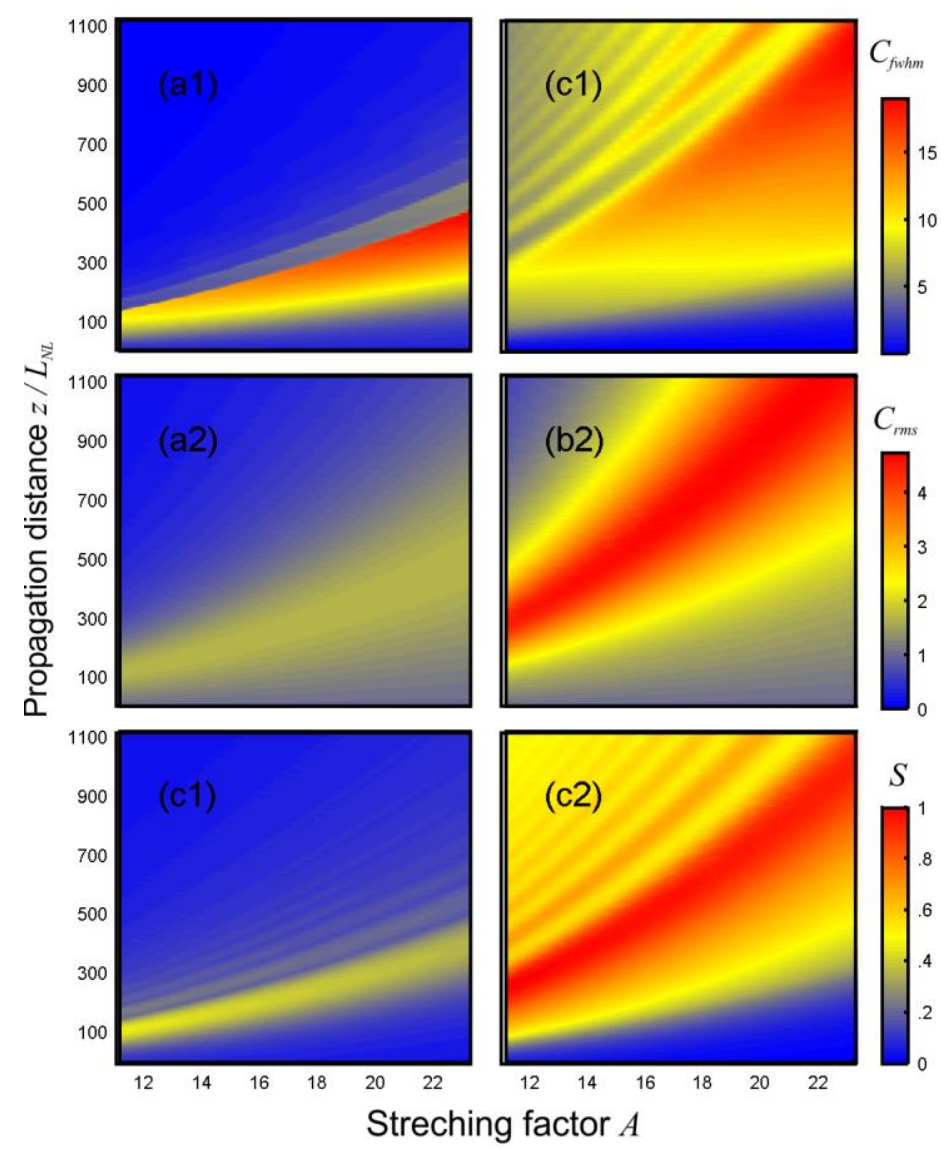

Figure 8 - Evolution of (a), (b) the FWHM and rms compression factors, and (c) the Strehl ratio versus stretching factor $A$ and normalized propagation distance for an initial Gaussian pulse propagating in a purely nonlinear fibre without (subplots 1 ) and with (subplots 2) phase correction.

\section{Conclusion}

We have presented a new, simple approach to enhance the spectral compression of negatively chirped pulses that occurs upon nonlinear propagation in a Kerr medium. We have numerically shown that in the nonlinearity-dominant regime of propagation, the intensity level of the spectral side lobes resulting from the mismatch between the initial linear chirp of the pulse and the SPM-induced nonlinear chirp can be efficiently reduced by an additional sinusoidal temporal phase modulation applied to the pulse. The required phase modulation can be readily accomplished by a lithium-niobate phase modulator or by employing cross-phase modulation with a sinusoidal signal. The proposed phase correction method enables notable improvement in the performance characteristics of the spectral compression process across a wide range of parameters that are readily available for experiments using picosecond pulses. For example, for an input picosecond pulse stretched by a factor 20 and with a Watt-level peak-power (after 
stretching), optimal spectral compression can be achieved after propagation in a km-long highly nonlinear fibre. The required modulating sinusoid would have a frequency of a few tens of gigaherts and a $\pi$ amplitude, complying with modulations routinely provided by telecommunications devices.

\section{Acknowledgement}

The authors would like to acknowledge support by the Région Bourgogne (Pari Photcom) and the Agence Nationale de la Recherche (Labex Action ANR-11-LABX-01-01).

\section{References}

[1]. Stolen R H and Lin C, 1978, Self-phase modulation in silica optical fibers. Phys. Rev. A. 17(4): p. 1448-1453.

[2]. Nishizawa $\mathrm{N}$ et al., 2010, Wideband spectral compression of wavelength-tunable ultrashort soliton pulse using comb-profile fibre. Opt. Express. 18(11): p. 11700-11706.

[3]. Tamura K and Nakazawa M, 2001, 54-fs, 10-GHz soliton generation from a polarizationmaintaining dispersion-flattened dispersion-decreasing fiber pulse compressor. Opt. Lett. 26(11): p. $762-764$.

[4]. Fedotov A B et al., 2009, Spectral compression of frequency-shifting solitons in a photoniccrystal fiber. Opt. Lett. 34(5): p. 662-664.

[5]. Zohrabian A V and Mouradian L K, 1995, Compression of the spectrum of picosecond ultrashort pulses. Quantum Electronics. 25(11): p. 1076.

[6]. Oberthaler M and Höpfel R A, 1993, Spectral narrowing of ultrashort laser pulses by self-phase modulation in optical fibers. Appl. Phys. Lett. 63(8): p. 1017-1019.

[7]. Planas S A et al., 1993, Spectral narrowing in the propagation of chirped pulses in single-mode fibers. Opt. Lett. 18(9): p. 699-701.

[8]. Washburn B R et al., 2000, Transform-limited spectral compression due to self-phase modulation in fibers. Opt. Lett. 25(7): p. 445-447.

[9]. Andresen E R et al., 2005, Spectral compression of femtosecond pulses in photonic crystal fibers. Opt. Lett. 30(15): p. 2025-2027.

[10]. Sidorov-Biryukov D A et al., 2008, Spectral narrowing of chirp-free light pulses in anomalously dispersive, highly nonlinear photonic-crystal fibers. Opt. Express. 16(4): p. 2502-2507.

[11]. Andresen E R et al., 2011, Transform-limited spectral compression by self-phase modulation of amplitude shaped pulses with negative chirp. Opt. Lett. 36(5): p. 707-709.

[12]. Rusu M and Okhotnikov O G, 2006, All-fiber picosecond laser source based on nonlinear spectral compression. Appl. Phys. Lett. 89: p. 091118.

[13]. Limpert J P et al., 2002, SPM-induced spectral compression of picosecond pulses in a singlemode Yb-doped fiber amplifier. Appl. Phys. B. 74: p. 191-195.

[14]. Fatome $\mathbf{J}$ et al., 2012, All-fiber spectral compression of picosecond pulses at telecommunication wavelength enhanced by amplitude shaping. Appl. Opt. 51(19): p. 4547-4553.

[15]. Bao C et al., 2015, Spectral compression of a dispersion-managed mode-locked Tm:fiber laser at 1.9 um. IEEE Photon. Technol. Lett. 28(4): p. 497-500.

[16]. Limpert J P et al., 2001, High-average-power picosecond Yb-doped fiber amplifier. Opt. Lett. 16(23): p. 1849-1851.

[17]. Korobko D A et al., 2016, Multistage fiber preamplifier comprising spectral compression for generation of high-energy laser pulses J. Opt. Soc. Am. B. 33(2): p. 239-245.

[18]. Boscolo S et al., 2012, Amplifier similariton fiber laser with nonlinear spectral compression. Opt. Lett. 37(21): p. 4531-4533. 
[19]. Kutuzyan A A et al., 2008, Dispersive regime of spectral compression. Quantum Electronics. 38(4): p. 383-387.

[20]. Finot C and Boscolo S, 2016, Design rules for nonlinear spectral compression in optical fibers. $J$. Opt. Soc. Am. B. 33(4): p. 760-767.

[21]. Agrawal G P. 2006 Nonlinear Fiber Optics, Fourth Edition, San Francisco, CA: Academic Press.

[22]. Perry M D et al., 1994, Self-phase modulation in chirped-pulse amplification. Opt. Lett. 19(24): p. 2149-2151.

[23]. Godil A A et al., 1994, Picosecond time-lenses. IEEE J. Quantum Electron. 30(3): p. 827-837.

[24]. Plansinis B W et al., 2015, Spectral changes induced by a phase modulator acting as a time lens. J. Opt. Soc. Am. B. 32(8): p. 1550-1554.

[25]. Kobayashi T et al., 1988, Optical pulse compression using high-frequency electrooptic phase modulation. IEEE J. Quantum Electron. 24(2): p. 382-387.

[26]. Varlot B et al., 2013, Experimental generation of optical flaticon pulses. Opt. Lett. 38(19): p. 3899-3902. 